\title{
KNOWLEDGE MANAGEMENT AUDIT - A METHODOLOGY AND CASE STUDY
}

\author{
Thomas W. Lauer and Mohan Tannin* \\ School of Business Administration, Oakland University \\ Rochester, MI 48304 - USA \\ lauer@oakland.edu tanniru@oakland.edu
}

\begin{abstract}
The strategic importance of knowledge in today's organisation has been discussed extensively and research has looked at various issues in developing knowledge management systems. Both the characterisation of knowledge and altemate models for understanding the acquisition and use of such knowledge have taken on significant prominence. This is due to the complexities associated with acquiring and representing knowledge, and the varied nature of its use in knowledge work. However, the role of the knowledge workers and the processes that guide their knowledge work as they meet the knowledge goals of an organisation have reccived little attention. This paper proposes a knowledge audit (an assessment of the way knowledge processes meet an organisation's knowledge goals) methodology to understand the "gaps" in the needs of a knowledge worker before one develops KM systems. The methodology also uses "process change" research to help build a socio-technical environment critical for knowledge work. The audit methodology is applied to a particular case and the implementation of the audit recommendations is discussed. Future implications of such an audit are also discussed.
\end{abstract}

\section{INTRODUCTION}

The argument for the strategic importance of knowledge in today's organisation has been made eloquently by a number of authors. For example, Drucker (1978) asserts that making knowledge work productive will be the great management task of the century, and that, "...the most valuable asset of a 21 st century institution... will be its knowledge workers and their productivity" (Drucker 1999, p.79). Others have noted the shift from an economy with a physical basis to one where knowledge and intellectual assets differentiate firms (Hansen et al. 1999). A corollary observation is that an organisation's ability to learn and acquire knowledge rapidly may provide the only source of sustainable competitive advantage (Winter 1995; Dierick \& Cool 1989; Lippmann \& Rumelt 1982). In a similar vein, changes brought on by technological development and global competition make rapid learning essential for the modern organisation (Croasdell 2001).

Given the wider recognition of the critical role "knowledge" plays in organisations, early research has focused on different aspects of knowledge management (KM). According to Ackerman (1996) information technologies enable automating organisational memory systems by 1 ) making recorded knowledge retrievable and 2) by making individuals with knowledge accessible. To develop computer supported knowledge management systems, earlier research has focused on knowledge management strategy (Davenport 1999), analysis and characterisation of tacit knowledge (Nonaka and Takeuchi 1995), technology support in design (eg. Duffy 2000), and social aspects of knowledge use and sharing in implementations (eg. Cook \& Brown 1999). Given the nature of knowledge and the complexities associated with its capture and dissemination, research in cognitive science, systems development methodologies, socio-technical systems, and technology diffusion have become antecedents to much of the research on the KM systems development. Specifically, these research streams are used to help us better understand the constituent components of knowledge and how they are acquired and used by knowledge workers.

One taxonomy of knowledge developed by Nonaka (1994) explicates the tacit and explicit dimensions of knowledge and is based on the earlier work of Michael Polyani (Polyani 1962; 1967) that has informed a significant body of research on knowledge based systems. An extension of this taxonomy was presented in Spender (1996) in which he added an individual and social dimension to the explicit - tacit distinction. For the individual, explicit knowledge is conscious whereas tacit knowledge is automatic. In the social realm, explicit knowledge is objectified. It refers to explicit policies and procedures. In contrast, tacit knowledge on the social level refers to the underlying organisational culture, the beliefs and values that are implicitly understood within the firm. One of the goals of the KM system is to make an individual's tacit knowledge explicit on both the individual and the social levels.

To help us better understand both the knowledge acquisition and use by knowledge workers, one model distinguishes between repository and network models (Ackerman 1996; Alavi \& Leidner 1999; Fahey \& Prusak 1998; Hansen et al. 1999). Alavi describes the two models as follows. "The repository model views knowledge as an object that can be collected, stored, organised, and disseminated. As such, these systems focus on managing explicit knowledge and primarily focus on the creation and storage/retrieval aspects of organisational knowledge management." Under this model, knowledge remains with the individual who possesses it and is transferred mainly through person-to-person contacts. Key technologies are databases and intranets and a critical issue for the implementation of these systems is the codification of knowledge. An alternative, network models do not, “... attempt to codify and extract knowledge 
from the individual who possesses it." Network models support KM processes that involve social interaction and direct communication among persons. This is in contrast to the repository model that involves person-repository and repository-person modes of knowledge transfer." While both models involve a socio-technical mix, the repository model throws its weight toward the technical system, while the network model emphasises the social system. In other words, the development of a socio-technical KMS has to recognise the existence of both these models in an organisation and address them appropriately.

The way in which knowledge is acquired and shared among knowledge workers is thus very complex and, hence, the knowledge work has more in common with work that is often associated with complex decision making than with operational or transaction-based work. Thus certain parallels may be drawn from the way in which information systems are developed to support complex decision processes. The development of decision or executive support systems often called first for an understanding the problem solving processes of a decision maker. Hence, before we embark on the development of KM systems, we need to understand the processes used by the knowledge-workers. Research has identified a few characterisations of processes associated with knowledge work [by Probst, Raub, and Romhardt (2000), Gupta and Govindarajan (2000)]. By understanding the key processes associated with knowledge work and their inter-relationships, one should be able to effectively position the KM system as one that support some of these knowledge processes. In other words, the focus of KMS development will be one of support for knowledge work, thus ensuring that the goals as well as implementation strategies are articulated in terms of how they are aimed at support a knowledge worker.

The focus of this paper is to conduct a "knowledge audit" - an a priori analysis of knowledge processes and the degree to which they address the knowledge goals of both the organisation and the knowledge worker. Just as "productivity goals" drive the development of transactional or operational systems and "decision quality goals" drive the development of a DSS, "knowledge goals" should drive the way knowledge is processed by a firm's knowledge workers. The deficiencies or gaps detected from such an audit can then lead to the development of a socio-technical knowledge management system that supports knowledge work using a mix of "repository" and "network" models. The next section will discuss the knowledge audit methodology and the third section will illustrate the use of this methodology to a particular organisation. Section four provides some recommendations and section five illustrates the changes made to the organisation since the audit recommendations were made. Section six provides some concluding comments along with some general observations on the audit procedure.

\section{A METHODOLOGY TO CONDUCT A KNOWLEDGE MANAGEMENT AUDIT}

An audit is typically intended to ensure that the goals of the organisation are met. If the goals of an organisation are to replace one system with another to gain efficiencies and the system is fairly structured, the audit procedure may view the system as a "black box" and "audit around the system" - compare the resources consumed and the reliability of the old and the new systems for viewing it from outside. If the system is complex, goals are somewhat illdefined, and reliability comparisons are difficult to make, then auditing may be performed by going "through the system" - understand the key processes and their reasonableness in meeting the qualitatively defined goals. The auditing of expert systems, for example, when the knowledge is intended to support rather than replace experts has followed similar knowledge process analysis and reasonableness tests [Agarwal, et. al, 1993]. The goal of the KM audit here is to understand the processes that constitute the activities of a knowledge worker, and see how well they address the "knowledge goals" of the organisation.

The knowledge processes discussed by Probst, Raub and Romhardt [2000] are shown in Figure 1 and these are used to perform the knowledge audit. It is top-down in that the knowledge goals provide the basis for evaluating various knowledge processes shown within the box. These processes are inter-dependent and consequently, one should not focus on one or a few of these processes to the exclusion of the others. Both knowledge goals and knowledge assessment are outside these knowledge processes practiced within the organisation. Knowledge assessment, similar to knowledge audit, is an evaluation of how well various knowledge processes are used to meet the knowledge goals. While an assessment can be frequent and internal to an organisation, an audit tends to be periodic and often driven by an external need. Results of the assessment can feed into potential modifications to the knowledge goals or activities within the knowledge processes. In the remaining section, each of the elements in Figure 1 is discussed in more detail.

\section{Knowledge goals.}

Knowledge goals give direction to knowledge management. In and of itself, the articulation of knowledge goals may provide a number of insights especially since this is likely to be a new activity for management. While the knowl- 
edge goals should support the broader organisational strategy, the two should be distinct given that the knowledge goals should be concerned exclusively with knowledge processes. Probst et al. identify three levels for these knowledge goals. Normative goals create knowledge awareness and a climate for sharing. Strategic goals define the organisation's core knowledge and identify skills that will be needed. Operational goals are concerned with implementing knowledge management methods and making normative and strategic goals realisable.

\section{Knowledge identification:}

This step calls for identification of the organisation's knowledge, which can be made known to members of the organisation on a need to know basis. Many companies find it difficult to maintain a map of internal and external sources of knowledge and information. Appropriate knowledge identification, known as knowledge transparency, enables members of the organisation to locate what they need without inefficiency or duplication of effort, and lessens the likelihood of uninformed decisions.

\section{Knowledge acquisition.}

Organisations can import knowledge from the 'outside'. Relationships with customers, suppliers, competitors, and partners hold the potential of providing knowledge. It is also possible to buy knowledge from various firms. In this process, we need to identify opportunities for knowledge acquisition and find out various external sources, from which knowledge and expertise should be sought.

\section{Knowledge development.}

Knowledge development focuses on developing new skills and knowledge internally. It is an activity that complements knowledge acquisition. It asks how can an organisation generate better ideas, new skills, better processes, and new products. Knowledge development includes all management efforts aimed at internally producing capabilities that do not currently exist in the organisation.

\section{Knowledge sharing and distribution.}

This process seeks to apply knowledge where it is needed. To do this, it is necessary to develop means for transforming isolated experiences into something the entire organisation can use. Given that it is not possible for anyone to know everything about everything, what can an organisation do to insure that knowledge of critical areas is gathered and shared where and when appropriate. An important activity is to understand how the transition of knowledge from the individual to the group takes place.

\section{Knowledge retention.}

A number of organisational activities take place that impede the retention of important knowledge, and these include the loss of an employee, mergers or acquisitions, reorganisations, or a failure to update and store knowledge of future value. There are a variety of means for storing organisational knowledge.

\section{Knowledge assessment.}

The way that knowledge management goals are established will directly affect the way they are measured. Methods for knowledge assessment must be tailored specifically to the organisation in light of the way its knowledge management strategy is formulated. Unlike areas such as finance or quality management, there are no set indices or measures for knowledge management. Some creativity must be used to establish means of assessment that will measure whether knowledge goals are fulfilled and how they contribute to the organisational mission.

The framework described in Figure 1 is general and action-oriented in that it enables the implementation of knowledge processes using a variety of tools and techniques, so that the knowledge goals are met. In addition, the application of the framework will help an organisation articulate its knowledge goals in concert with its overall organisational strategy as well as support for knowledge work, and lead to the development of criteria for measuring success. Given that the knowledge management activity is articulated as a set of processes that are used to reach a set of stated goals, the knowledge audit then looks at how well each of these processes address the goals. 


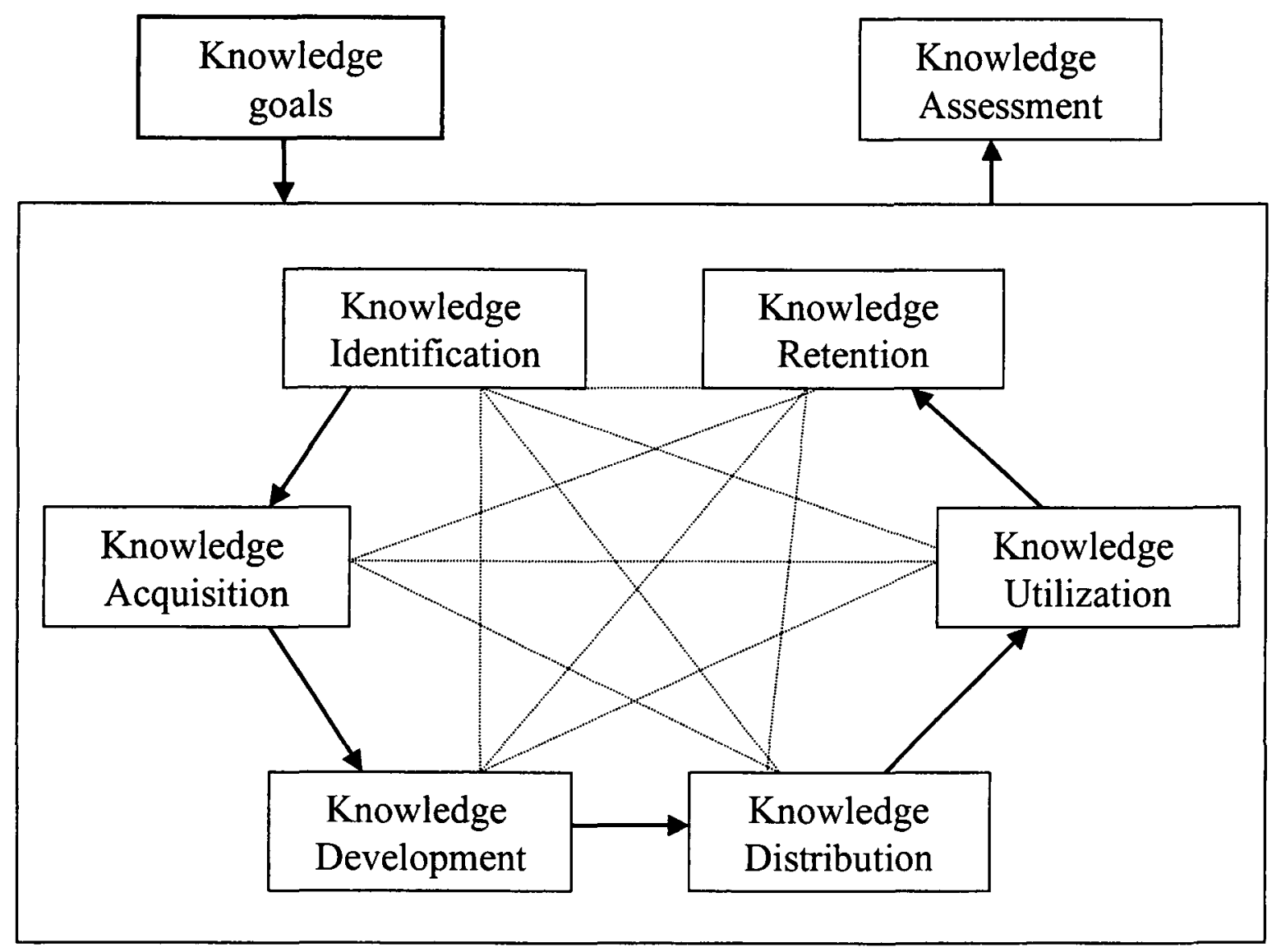

Figure 1 Knowledge Management Framework

The challenge for an organisation is to design a socio-technical system that will enable knowledge workers to be effective in performing various knowledge processes within the organisational context. Levitt (1972) describes a model (see Figure 2) that emphasises the importance of four related factors in designing a socio-technical system and these include, in addition to the task, the technology, organisational structure, and people. So, the knowledge audit needs to take into consideration the relationship of the current knowledge processes to the organisational structure, people and technology, before developing recommendations for process change. In a later section, the same model is also used in recommending process changes.

This, hence, leads to a methodology for conducting a knowledge management audit. 


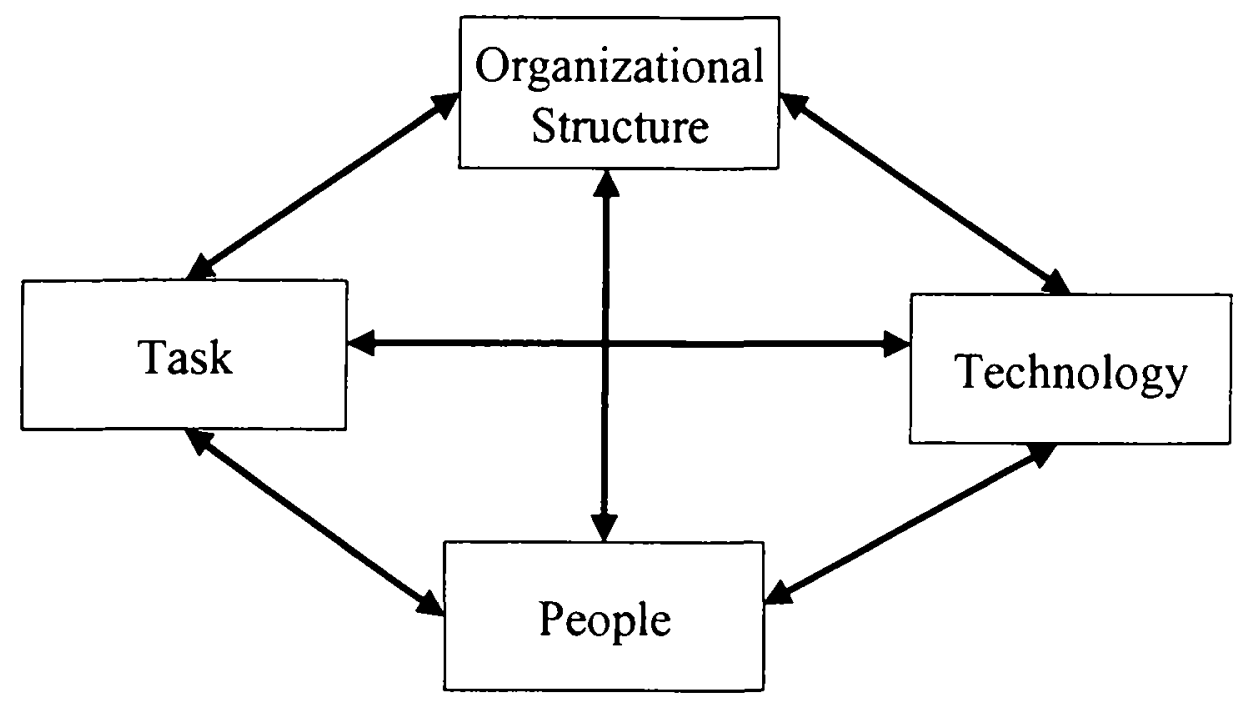

Figure 2. Process Change Model

\begin{abstract}
A Methodology for Knowledge Management Audit
Articulate knowledge management goals, which include normative, strategic, and operational goals.

Describe each knowledge process (shown in Figure 1) and its relationship to the organisational structure, people and technology (socio-technical environment).

Assess how well each process in this environment is fulfilling the knowledge goals articulated in step 1.

Next section will discuss an application of this audit methodology to an organisation.
\end{abstract}

\title{
KM AUDIT OF ATIB ORGANIZATION
}

Applied technology in business (ATiB) program is established by the school of business administration at a midwestem university. Business students, who may come from any major are competitively selected to participate in this program and, upon completion, will be awarded an ATiB minor. The program's primary mission is to enable the students to gain the skills they need to apply information technology to solve real world problems that are provided by the program's corporate sponsors. The students are the "knowledge workers" that use "corporate projects" as the primary vehicle to learn and apply many of the skills they acquire in the classroom related activities. In effect, "corporate projects" act as the key source of knowledge that is shared by the three primary stakeholders (students, faculty and industry). Given the central role these projects play, this "project knowledge" becomes a key success factor. Even though the ATiB program is established within an educational setting to support student learning, its need to provide "project knowledge" for workers and retain as much of that knowledge as possible before the students leave the program poses many interesting challenges that can be generalised to many industrial settings.

The ATiB organisation had finished its third year, completed 150 projects, and graduated 34 students when the initial audit was performed. Since that time, the program has completed 50 additional projects and graduated 16 more students. Figure 3 illustrates how the knowledge workers (students) interact with various knowledge providers (director, faculty/staff, project sponsors, seniors and other students) to obtain "project knowledge" that is needed to complete their projects. The ATiB laboratory plays a crucial role in supporting the knowledge workers. This layout is shown in Figure 4. The lab acts as a meeting place for many formal activities (such as workshop presentations), informal get-togethers and team meetings of students. In a way, the lab acts as a social setting for bringing many knowledge management activities together.

The organisational structure is flat. All the students (junior and senior), faculty, and sponsors have a direct and open line of communication to the director. Faculty and director together provide coordination on corporate projects from the academic side and a corporate sponsor provides coordination of the project from the industry side. Senior students also coordinate and/or mentor projects of the juniors. 


\section{Knowledge Goals (Normative, Strategic and Operational)}

At the time the knowledge audit was initiated, ATiB had not defined any of its knowledge goals. The following represents a first cut at inferring both the normative and strategic knowledge goals for ATiB. In order to support the program's mission [develop adaptive, technology literate, innovative business professionals], the knowledge organisation, has established the following normative goals:

1. Create an inter-disciplinary orientation where the student (knowledge worker) develops a respect for diverse knowledge rather than viewing knowledge from the standpoint of a single 'functional silo'.

2. Create an organisational environment where constructive sharing of knowledge and cooperation are practiced in support of the ATiB organisation.

3. Create a learning culture that encourages seeking new knowledge and sharing it with others.

Inter-disciplinary orientation The strategic goals that support the creation of an inter-disciplinary orientation are:

Recruit students from multiple functional backgrounds and interests into a common program that has a single mission - effective utilisation of IT.

Form teams with students from multiple disciplines to solve problems of all types posed by corporate sponsors from different industry.

Select projects that require students to draw upon diverse knowledge.

Use case studies and class discussion to reinforce the need to solve problems with an organisation wide focus as opposed to functional focus.

Organisational orientation The strategic goals that support the creation of an organisational orientation are:

Engage students in activities and put them in positions in support of the organisation's objective of supporting each one's learning and meeting sponsor needs.

Create opportunities for each one to present their views and discuss their projects and challenges in a forum that encourages constructive dialogue and discussion.

Facilitate ease in sharing of knowledge, thus giving each one an opportunity to act as expert in some areas in support of all the others.

Learning culture The strategic goals that support the creation of learning and knowledge culture are:

Recruit program staff that recognises the need for constant learning and that have a willingness to share what they know with others.

Encourage learning from failures and successes by supporting the sharing of lessons learned with others.

Encourage mutual support and remove any inhibitors for individual growth.

\section{Mapping of Knowledge Processes to Program Activities}

The program-related activities are mapped to the knowledge processes (shown in Figure 1) in Table 1. One noteworthy result is the absence of any program-related activities for knowledge acquisition. Here, the knowledge acquisition refers to purchasing or otherwise acquiring knowledge from outside the organisation. Since the main focus of the ATiB program is the development of students' skills and knowledge, there would be little if any knowledge acquisition. 
Table 1.

ATiB Activities addressing the Knowledge Proc-

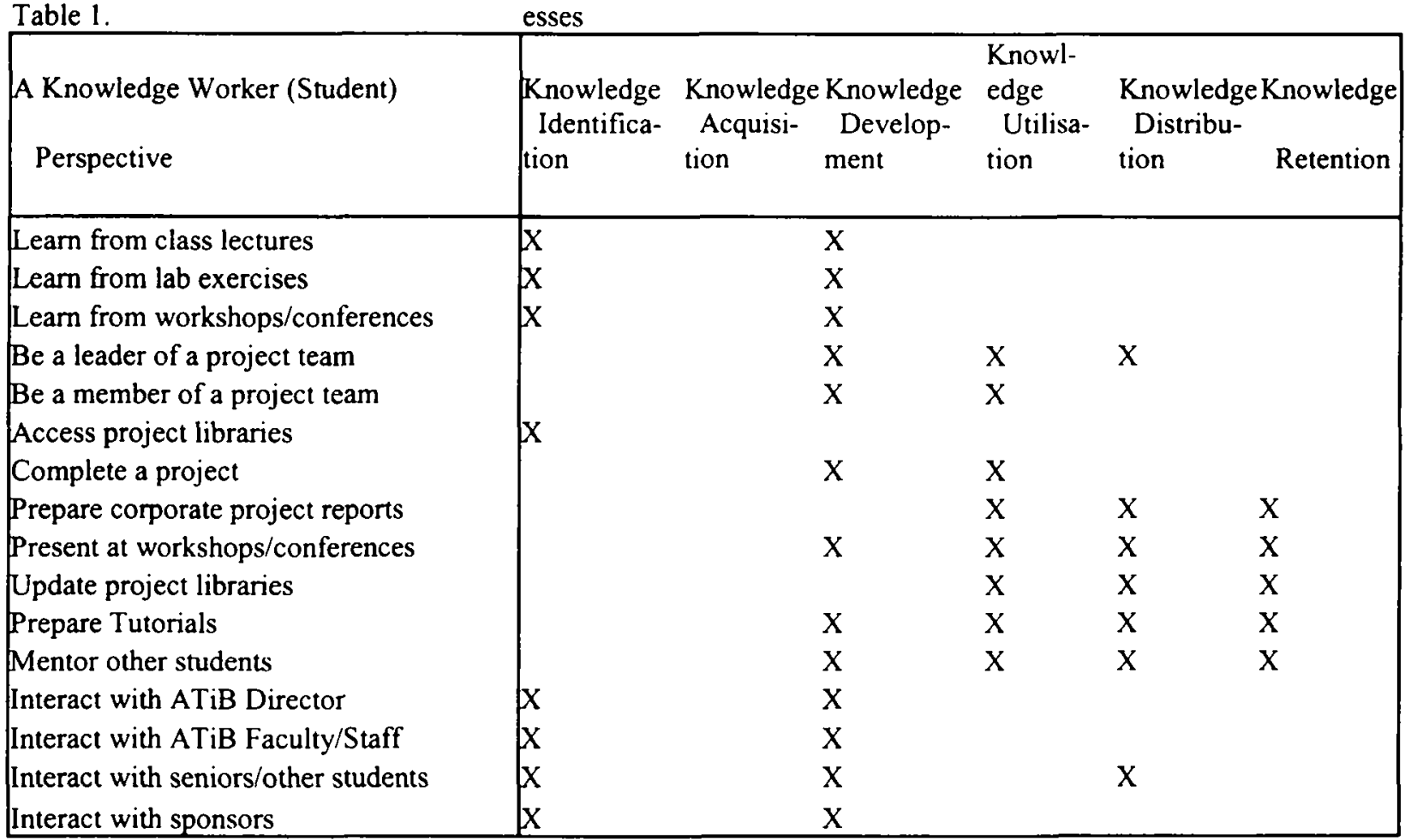

One of the authors used graduate students from a KM class he taught to conduct a knowledge audit of ATiB as a class project. The students were required to interview various ATiB stakeholders (including students, former students, faculty, and corporate sponsors) in order to understand how ATiB students performed the knowledge processes discussed previously. Students were organised into teams to conduct the interviews in three phases. Each student team consisted of four members. Each interview was conducted by two students, one who asked questions, and the other who served as a scribe. The interviewers used e-mail for follow-up questions. During the first phase, a student team interviewed the Director to determine normative, strategic, and operational goals. The second phase covered knowledge identification, acquisition, and development. The third phase covered knowledge distribution, utilisation, and retention. Interviewees for the second and third phases included the Director, current students, former students, faculty, and corporate sponsors. What follows in this section is based on the results of the final report from the graduate KM class.

\section{Audit Observations}

Knowledge Identification

Much of the knowledge needed to complete a project is identified on an ad hoc basis (as dictated by the project), and the director, faculty and senior students play a significant role in this process. The physical layout and the flat organisational structure support a great deal of informal interaction that often facilitates serendipitous knowledge discovery. In addition, there are a number of functions that take place during the year that serve the dual purposes of socialising the students and familiarising them with the work of other student teams and the sponsors' business. In spite of the fact that the role of technology is somewhat limited, the culture permits networking and mutual project support for students seeking the needed knowledge from others.

Knowledge Acquisition

As noted previously, knowledge acquisition plays almost no role for the ATiB students since it refers to acquiring knowledge and skills from outside the organisation. 
Knowledge Development

While this process of the KM framework is well developed, the dynamics of the field (IT) and the diversity of projects makes the knowledge content incorporated into the program somewhat fluid. The role of faculty in the knowledge development process is not well articulated today, and the role of technology (such as the use of self-paced training material) is not well integrated into the program. Also, the program today relies on the self-motivation of the knowledge workers and the requirements of the corporate projects for the overall knowledge development process. In the long run, a set of metrics are needed to ensure a pre and post assessment of the knowledge developed by the students.

\section{Knowledge Utilisation}

Again, as in the development phase of $\mathrm{KM}$, the degree to which a student utilises his/her knowledge is dependent on the project they are asked to complete. Given that there is no scientific way the projects are assigned to students today, the degree of knowledge utilisation (other than general project management knowledge) varies with each student.

\section{Knowledge Distribution and Sharing}

The role of technology for knowledge distribution is somewhat limited to storing status reports, email communications, PowerPoint ${ }^{\mathrm{TM}}$ presentations, etc. While these provide a way to distribute knowledge to those in the future, it is not well indexed for easy search and dissemination. Also, there are not many opportunities for abstraction and generalisation.

\section{Knowledge retention}

While the mentorship program is an effective means of distributing knowledge from seniors to juniors, there is no concerted effort to preserve much of this knowledge in a static form that can be used by others. For example, there are no de-briefing sessions for seniors leaving the program. Technology has not been incorporated as a means of storing and indexing lessons learned in a searchable format. In addition, it may prove to be useful to categorise other project documentation with sub-categories relating to features of the project, business or information technology. In order to support such a change to the technology for knowledge retention, it would be necessary to modify the affected parts of the way that projects are carried out and to insure that student inputs to the database were part of their evaluation.

\section{Seeking synergy between knowledge goals, processes and socio-technical environment (steps 1 and 2)}

One of the critical aspects of a knowledge management audit is to identify disconnects between the socio-technical environment, the generic knowledge processes, and the knowledge goals. In this way, the audit points to changes in the socio-technical environment and the generic knowledge processes needed to support the knowledge goals in a way that is very similar to creating a change management culture that sustains process redesign effort for radically improving corporate performance during process redesign. Both the knowledge sources and organisational structure (see Figures 3 and 4) and the discussion above, highlight a few major program attributes (listed below) of the sociotechnical environment of the ATiB program that, we believe, will continue to support the knowledge processes and their meeting the stated knowledge goals:

Support of open interaction among students as well as among all the program's constituents: faculty, sponsors, alumni and students, using a variety of informal settings for communication and sharing of knowledge; Support of new technology exploration without fear of failure and sharing that knowledge with no perceived threat of losing competitive edge (as evaluation is based on project success and individual learning rather than an evaluation on a curve);

Recruitment of students from multiple disciplines and assigning them to projects with diverse scope and coverage; and

Recruitment of sponsors from diverse industry to ensure that business and technology changes will continue to infiltrate into the program and keep it current and learning oriented. 
Next section will provide a summary of the audit observations and make recommendations for changes in the knowledge processes.

Figure 3. A Know ledge Worker Perspective

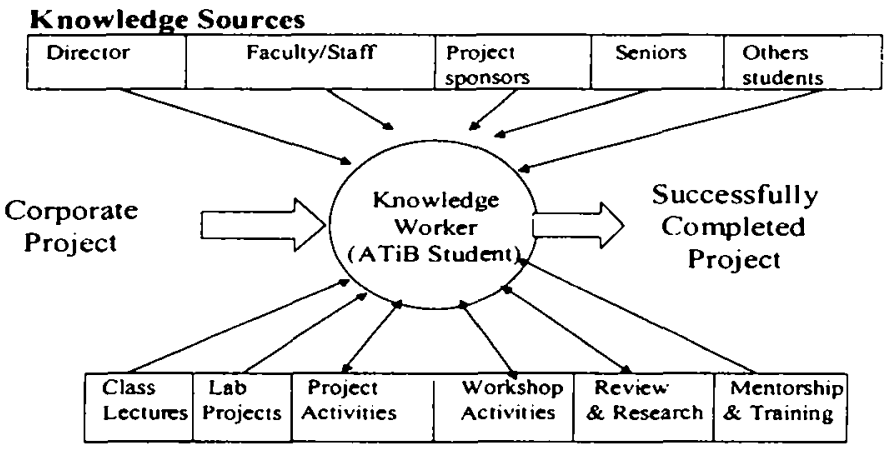

Knowledge Worker Activities

\section{Figure 4. Physical Layout}

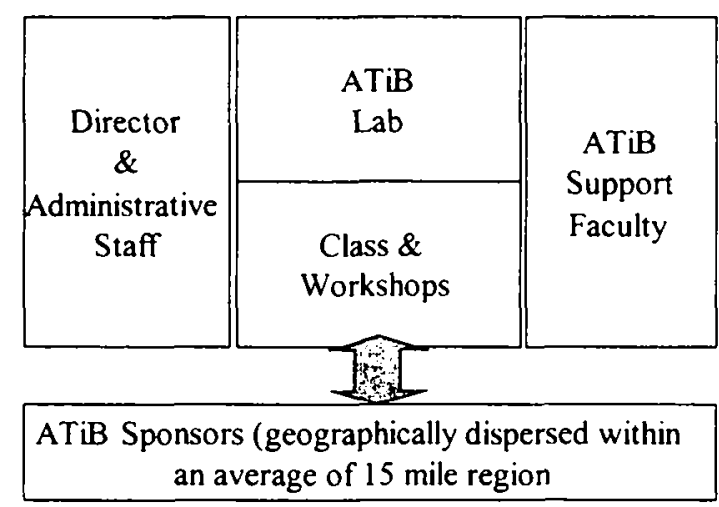

\section{RECOMMENDATIONS FROM THE KM AUDIT}

Overall, the results of the KM audit can be summarised within three categories: with respect to knowledge strategy, within the social system, and within the technical system. From a KM vantage point, ATiB's social system functions well. Consistent with this observation, ATiB relies on a network model in that knowledge is regarded as resting with the individual and is transferred primarily through person-to-person interchanges. The audit suggested weaknesses within the technical system and with regard to unarticulated knowledge goals. The audit suggested opportunities to use technology for knowledge development, knowledge use, knowledge sharing, and knowledge retention. Prior to the audit, ATiB had not explicitly considered knowledge goals. Consequently, it had not made knowledge goals integral to its stakeholders and its day-to-day activities. Since the audit was based on an analysis of generic knowledge processes, changes aimed at improving them should be considered in light of research on "process change".

Process reengineering methodologies emphasise the importance of aligning information technology, organisational structure, job design, rewards and controls with the changed process. (See eg. Hammer \& Champy 1993, Kettinger et al. 1997, Klein 1994, Rummler \& Brache 1995). The importance of organisational culture and organisational values as part of the common ground for change is also noted by some of these methodologies. However, as Davenport et al. (1996) note, there are significant challenges in applying traditional approaches to process change to knowledge work and knowledge workers. The challenges they report include: a) the variety and uncertainty of inputs and outputs, b) the unstructured and individualised nature of the work, c) the lack of clear separation among inputs, process, and outputs, d) the lack of measures of process effectiveness, e) worker autonomy, $f$ high variability in performance across individuals and time, and g) lack of information technology support. In spite of these difficulties, there are 
important features of reengineering methodologies that can be incorporated into a methodology for the analysis, design, and implementation of a knowledge management system.

The emphasis on harmonising the social and the technical system is an important feature of BPR methodologies, and this may be carried over to knowledge management. However, the strong process centric orientation may be less suitable due to a number of differences between successful knowledge management projects and the typical successful BPR project [Davenport et al. (1996)]. Differences they noted included the absence of ambitious stretch goals for performance improvement and new designs that focus on features rather than actual processes. BPR methodologies based on the model described in Figure 2 identify process (task) as the primary focus of change, and identify technology (usually IT), organisational structure (how communication and reporting occurs), and people (job design, including rewards and training) as infrastructure factors, which must be addressed as part of this change. In other words, if a process is changed without complementary changes to these infrastructure areas, the process change will be ineffective. Given the difficulties of process change in general, and knowledge process change in particular, a cautious incremental approach was favoured over attempting to institute radical change. Changes were to be based on the following three recommendations.

\section{Three major recommendations}

The audit shows that the knowledge organisation has been operating relatively successfully using a "network model" of informal links among various stakeholders such junior and senior students, sponsors, faculty and alumni to complete many of the knowledge processes, and the role of the "repository model" is relatively minor. While this alone is not inappropriate for a small organisation, over a longer period of time, as the knowledge base increases in size and the knowledge resources (people) move far from the knowledge organisation, a more formal "repository" model may be relevant even if the network model continues to play an important role in the success of the program.

Second, since the knowledge organisation has evolved over its three years (at the time of the audit), many of the goals, strategies, etc. have been evolving as well. While this is not atypical for a new organisation, these need to be articulated and disseminated effectively to various stakeholders. Such a dissemination allows for a stakeholder understanding of the knowledge goals/strategies and their potential input in any refinement to these goals/strategies as the environment changes. This can also support an eventual distribution of some of the roles and responsibilities of running this organisation to others.

Lastly, while the program appears to be successful in accomplishing its goals, many of the comments related to these accomplishments are anecdotal. More formal metrics have to be developed and instituted to ensure that the accomplishment of goals, quantitative as well as qualitative, are tracked over time. Next section will discuss the implementation of the three audit recommendations.

\section{IMPLEMENTATION OF AUDIT RECOMMENDATIONS}

\section{Repository Support of Project Knowledge}

An ATiB Portal was developed and implemented in May-August 2000 (four months after the audit) and several of its features are shown in Figures 5-11.

Portal Features: Figure 5 provides a list of features that are available to the administrator, which include adding new sponsors, defining projects, assigning students to sponsors, etc. Some other administrative features such as assigning grades, providing access to the portal to guests and sponsors, etc. are also included. 
Figure 5 Opening Panel and Portal Features

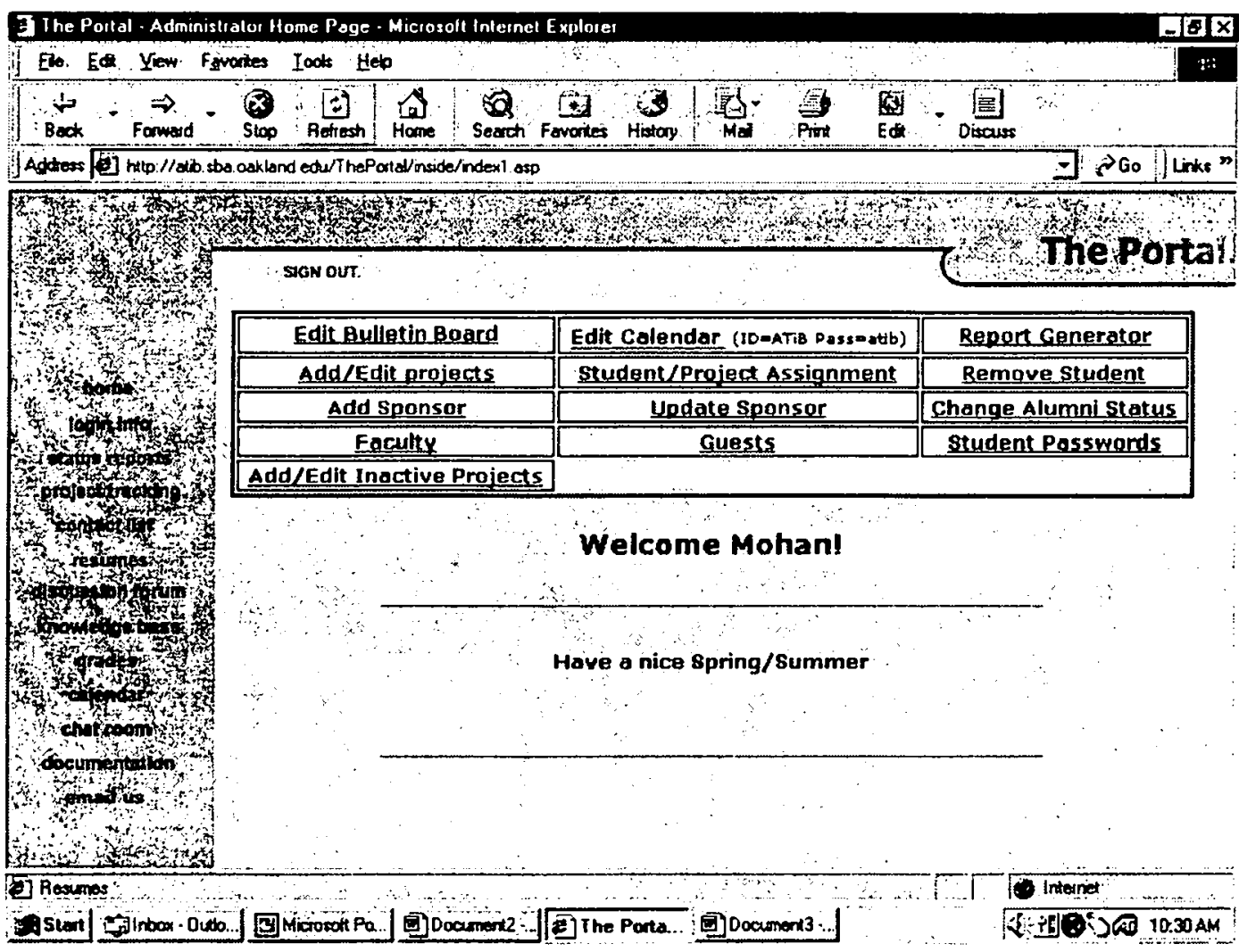

Project Status Reports: Figure 6 provides a screen that lists status reports of all projects that are under execution in any given semester and by highlighting one of these, the students as well as a sponsor can get access to specific activities that have been completed under the project in all the weeks since the project started. These status reports are moved to archival storage at the end of each term and become a part of knowledge base, which can be accessed by students at a later time. Effective search mechanisms, besides the name of the corporation or team-leader are being explored. Figure 7 provides a sample of the detailed status report for a week and Figure 8 provides the project tracking report, ie. where a student is on a project based on a set of steps that were a-priori identified. 
Figure $6 \quad$ Project Status Reports

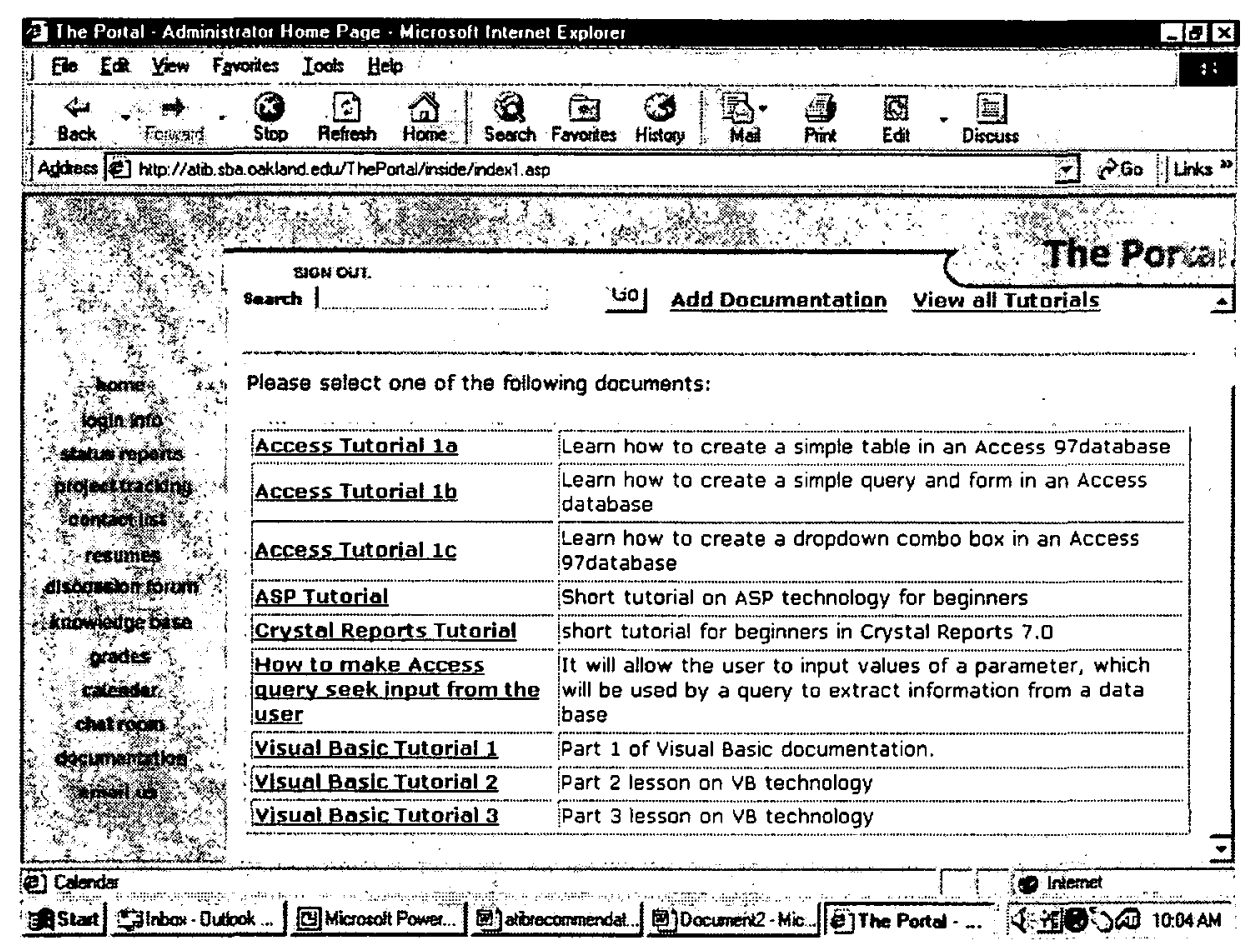

Figure 7 Details of a Status Report associated with a Particular Project

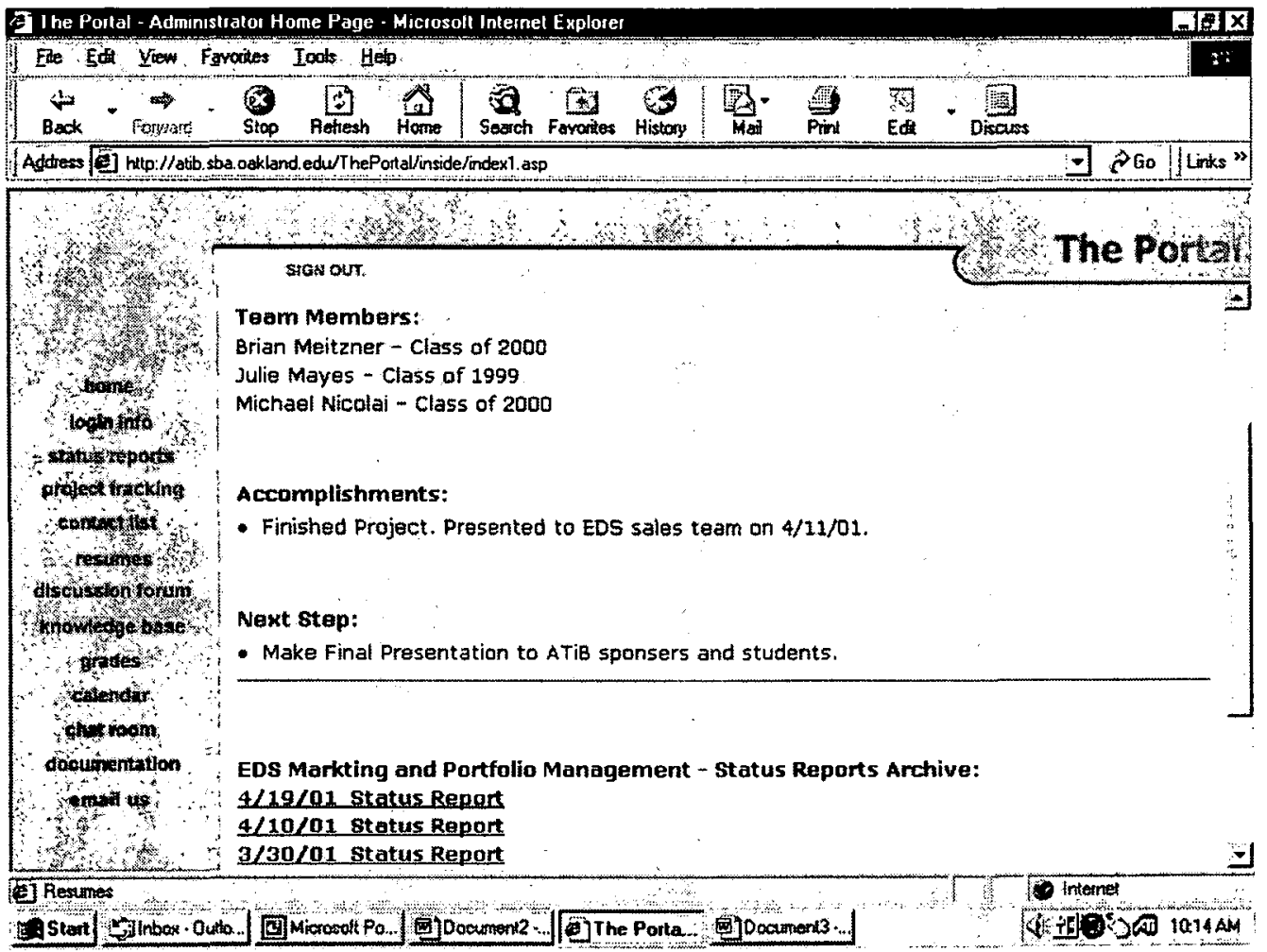


Figure $8 \quad$ Project Tracking

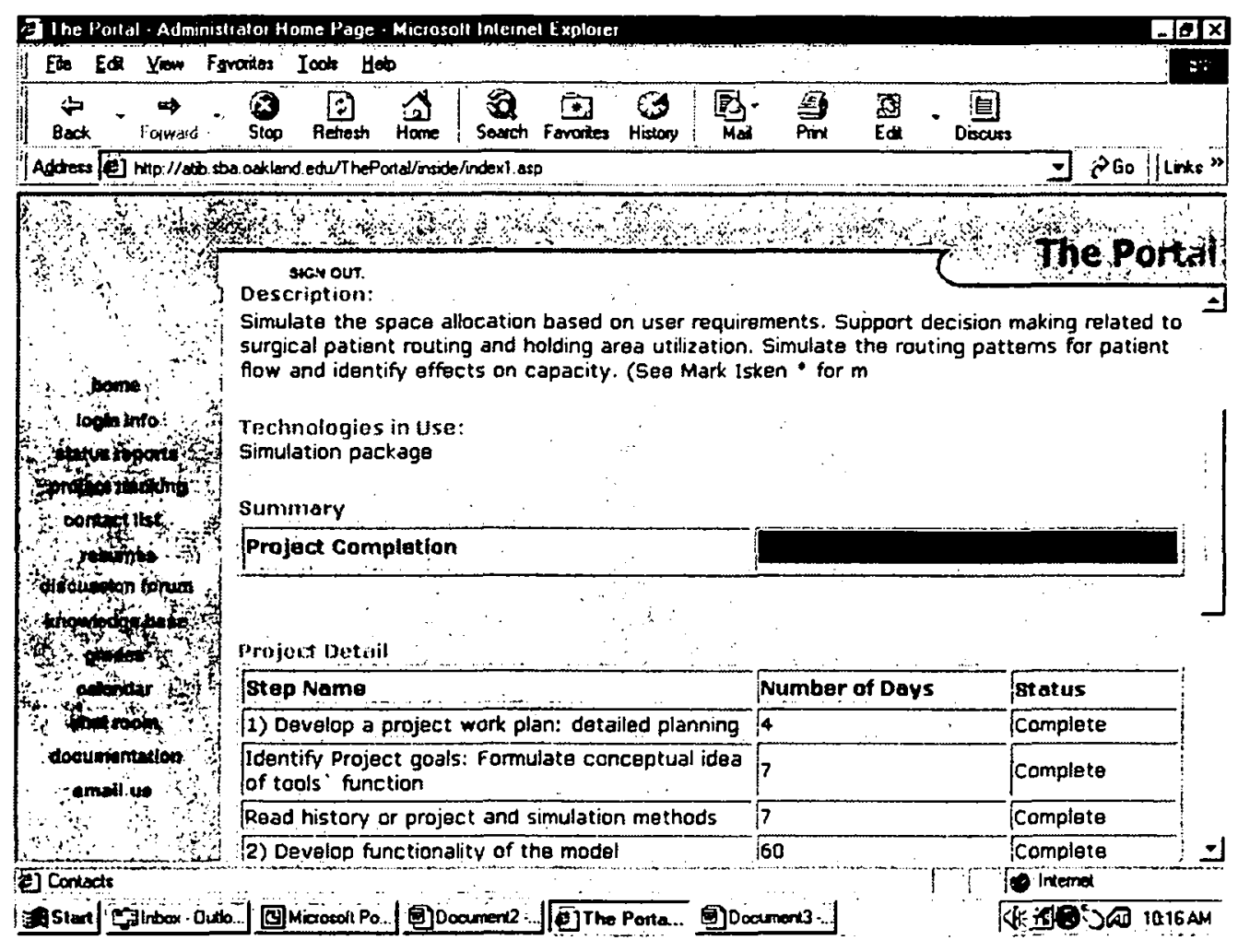

Documentation: Figure 9 provides a list of tutorials and special reports (including past project summaries) that have been developed each term. These can form the basis for self-paced learning and act as reference documents to refresh one's knowledge about a technology when it is called for. 
Figure 9 Documentation

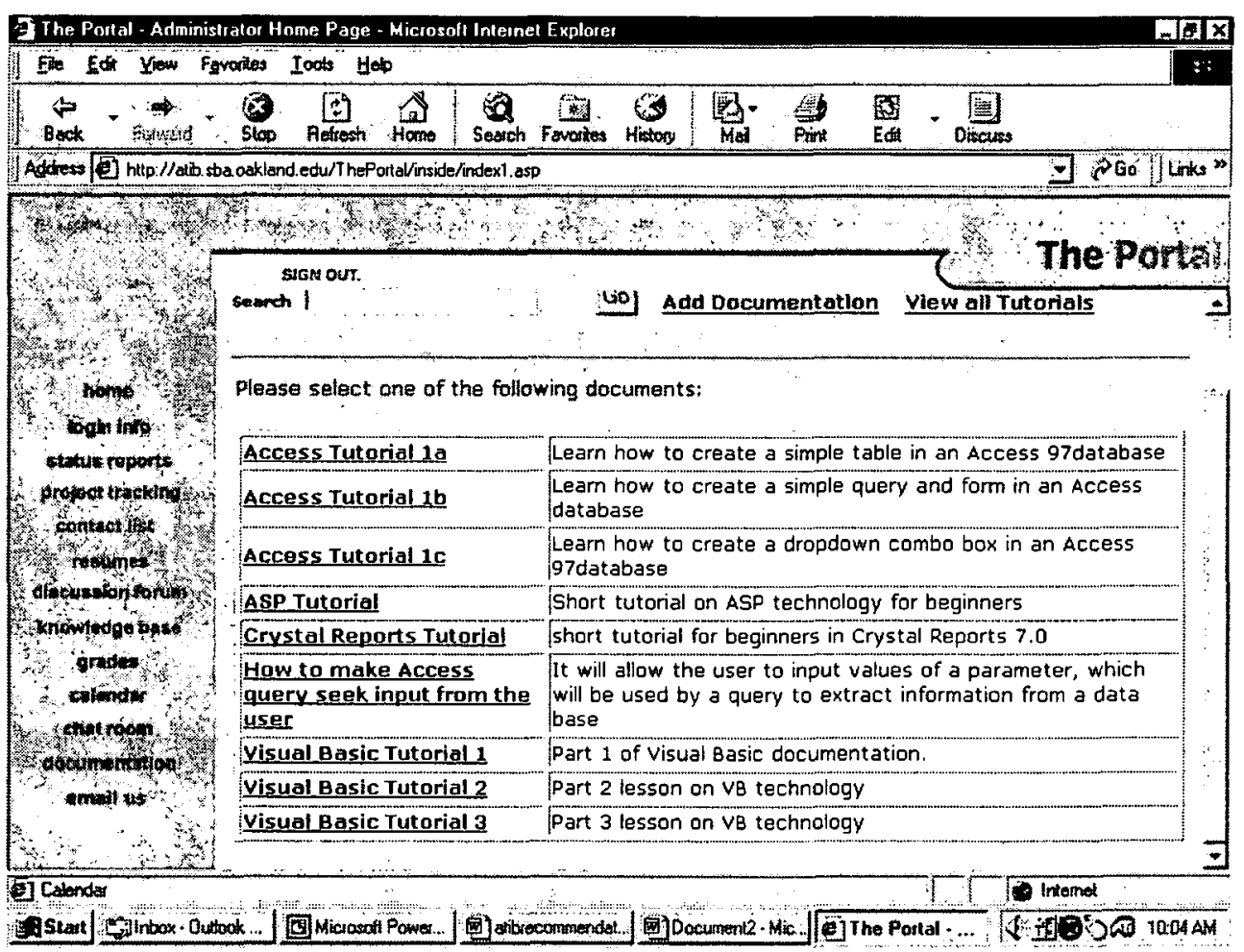

Knowledge Base:

Figure 10 provides a list of projects that are being worked on for a particular sponsor. This information is used to get a general idea on the type of projects a firm may engage in, especially if a new sponsor is looking to define projects in an area similar to theirs for potential students. 
Figure $10 \quad$ Knowledge Base

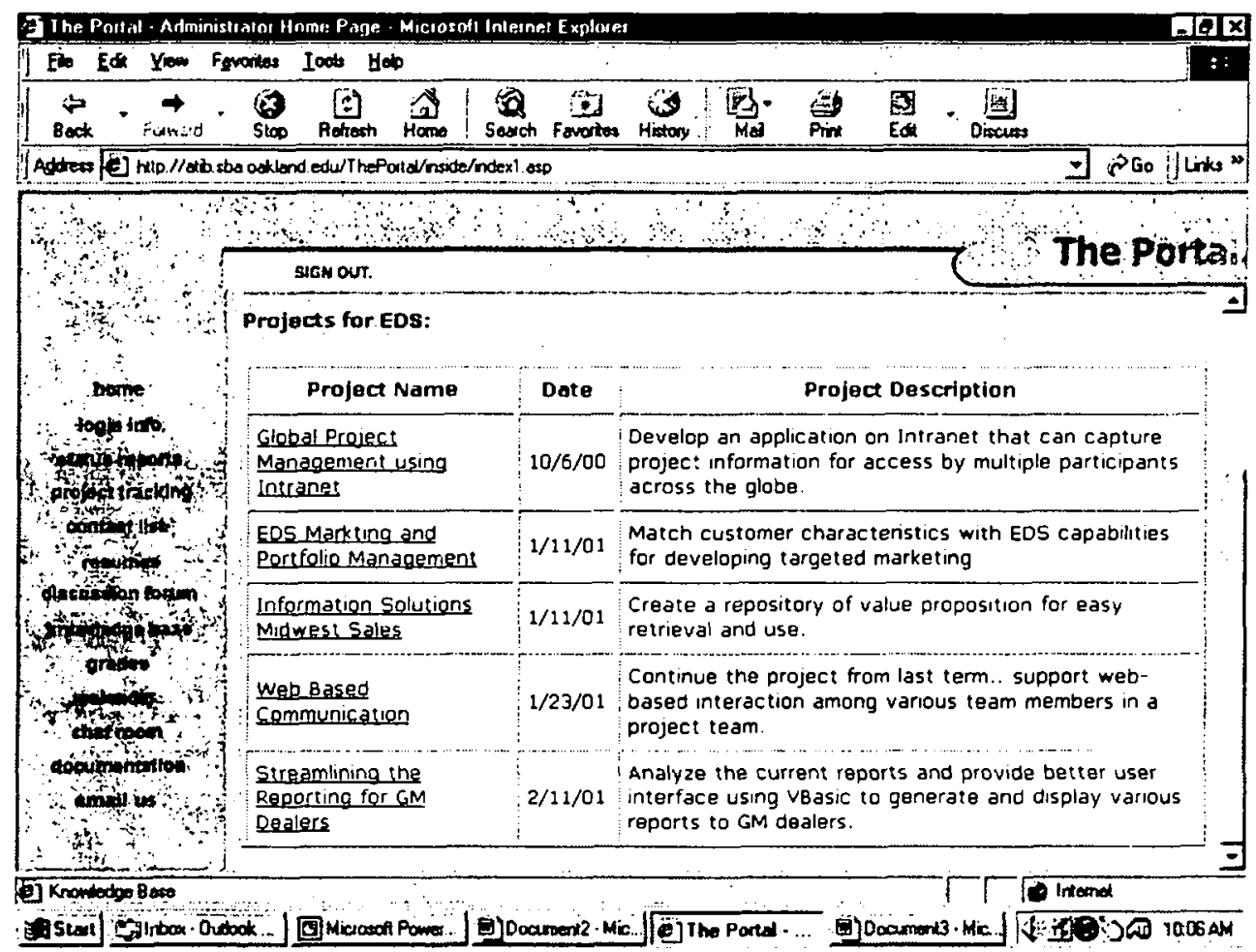

Discussion Forum Figure 11 provides a discussion forum that can be used by students and sponsors to ex-
change ideas on a topic of interest. Questions can be posed by a student and can be answered by alumni, seniors or sponsors that have specific knowledge on the topic.

Note that not only does the discussion forum provide support for internal communication but it also supports one of the key features of the ATiB program: networking students with alumni and sponsors. The discussion on an advanced program in Figure 11 is an exchange between a student, alumni and a sponsor. In addition to these features, the portal also provides a contact list that includes email, phone or fax, resume information, etc. 
Figure 11 Discussion Forum

Julie is a student, Peggy is a alumni and John is a sponsor

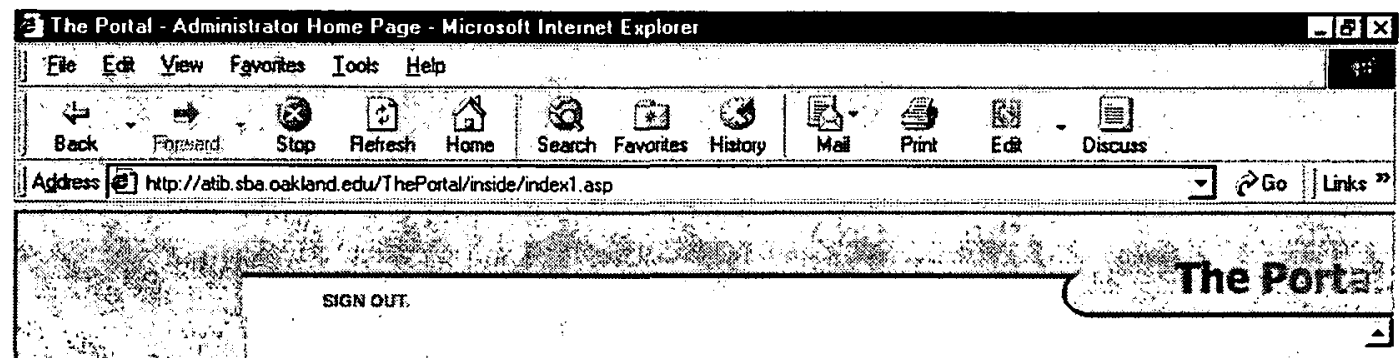

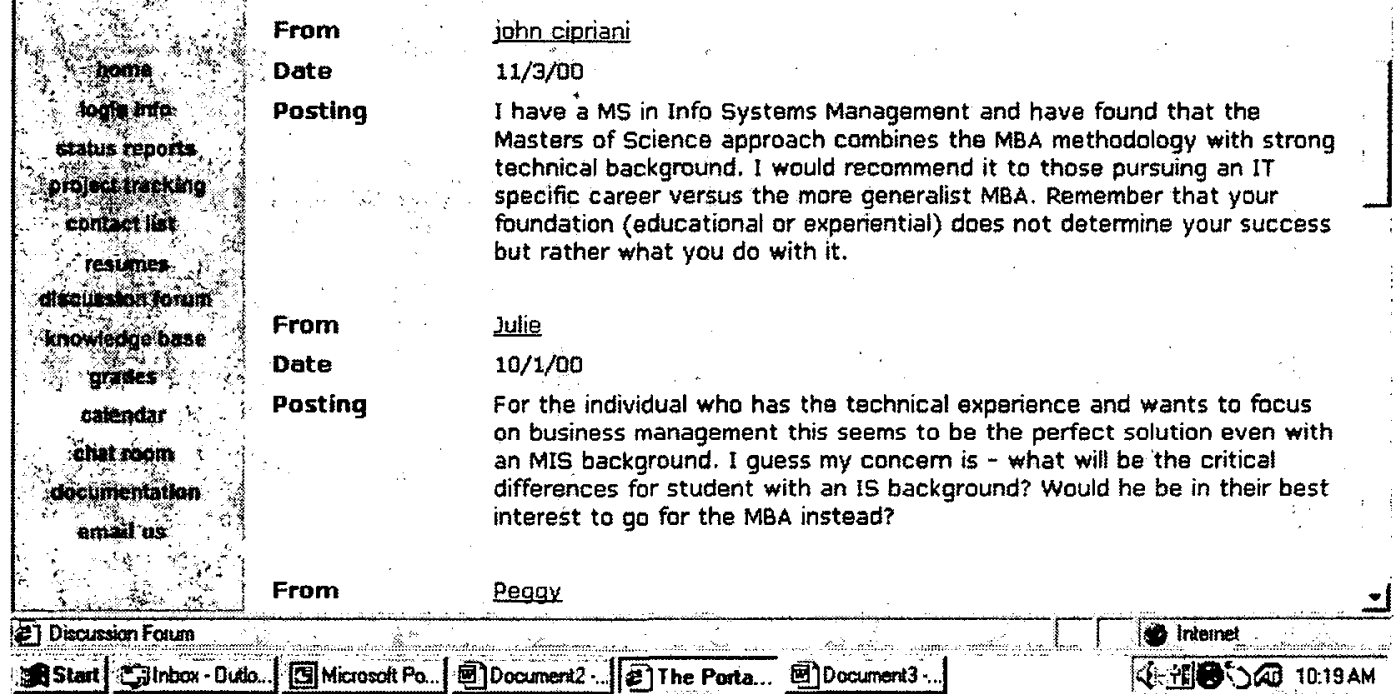

\section{Streamline Articulation and Dissemination of Knowledge Goals and Strategies}

In an evolving organisation, some of the goals and strategies may be initiated by those in-charge. Even so, it is critical that these are shared among various stakeholders for review and refinement. This may enable the eventual redistribution of some of the roles and responsibilities of the organisation to others and the creation of some degree of redundancy to mitigate some of the risk to the organisation. A web-based framework was developed in April 2001 and will be used to communicate many of the program's goals, strategies, and processes to various stakeholders to get their reaction and increase their involvement. Figure 12 provides a sample framework that is used to highlight various features of the program and its stakeholders, and the matrix provides hyperlinks to dissemination status, responsibility assignment, etc. 
Figure 12 ATiB Continuous Improvement Accreditation Process - Project Features and Stakeholders

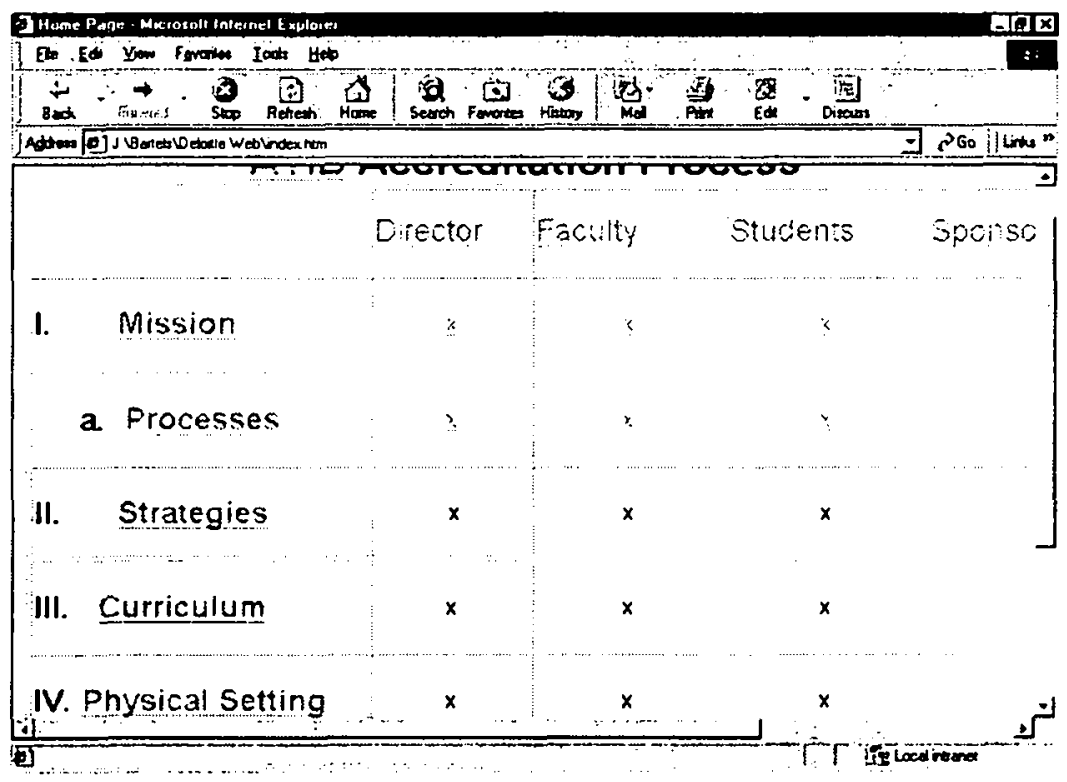

\section{Development of metrics}

Since some of the strategic goals call for inter-disciplinary orientation, learning, and knowledge sharing, measuring the degree to which an individual knowledge worker has accomplished these goals is difficult to measure. While many of the strategic and operational goals are intended to support these normative goals, one still needs feedback mechanisms (formal as well as informal) to gather such information after a student leaves a program. Three different mechanisms have been put in place to gather the needed information at different levels of granularity.

First, a project survey instrument was developed and is now sent to students at the end of each project to assess their perception of success and to ascertain what features characterise the project (problem complexity, team maturity, faculty and sponsor coordination, etc.). The aim of the survey is to evaluate the degree of impact different project features have on project success and ultimately to influence the manner that students are selected for projects. A similar survey is now sent to corporate sponsors as well. Second, a formal survey was developed and will be sent at year-end to various alumni and sponsors. Its purpose is to determine their level of satisfaction with the pro$\mathrm{gram} /$ projects in meeting their expectations. The survey is administered to get students' reactions after they leave the program and to measure long-range effects of the program on students' careers and sponsors' projects.

Third, a formal albeit qualitative forum has been established as a part of a yearly conference, enabling various alumni to reflect on their collective experience since they left the program and the way their current work relates to experiences in the program. This is yet another way to understand the role of the knowledge sharing and learning culture that was embedded in the program on a student's career in the long run. In summary, the knowledge audit conducted one year ago has highlighted several important and critical aspects of ATiB as a knowledge organisation. Some aspects, the effective use of the network model and the cross-disciplinary culture, have contributed to the overall success of the program. It, however, has highlighted other measures the organisation should undertake to streamline various knowledge processes to support both repository related activities and continual assessment. Many of these recommendations have been put in practice and a future audit may be used to assess their impact.

\section{DISCUSSION AND CONCLUSION}

Some specific probes that have provided motivation for developing KM systems in the past include the acclamation of new knowledge workers and mitigating the loss of intellectual capital through the attrition of key knowledge workers. By thinking of ATiB more broadly as a knowledge organisation, we gained insights into these two related problems. In addition, by conducting the knowledge audit, we are able to offer some observations concerning methodological issues: the usefulness of knowledge audits for establishing knowledge requirements, and the relationship between network and repository models. 
Acclimating a new knowledge worker to an organisation. ATiB is quite successful in its efforts to introduce new students to its program. The design of training experiences so that responsibility and independence are incrementally shifted from external sources such as faculty and sponsors to the student is a key factor in developing technology capable project workers. The use of seniors in a mentorship role with juniors is vital to the success of this incremental approach. ATiB supports productive social interactions through the physical design of the working space and through holding a number of quasi-social functions throughout the year. ATiB makes extensive use of workshops and conferences where students can share the results of their work with other students, faculty, and sponsors. Firms interested in instituting knowledge management programs could incorporate such techniques. It can be socially rewarding, personally satisfying, and intellectually valuable to present the results of a successful project to one's peers. Lessons learned conferences can serve as a means of preserving organisational knowledge while simultaneously enabling the development and strengthening of interpersonal networks.

Planning for the loss of critical intellectual capital. For ATiB stakeholders, thinking of itself as a knowledge organisation where the graduates represented a loss of intellectual capital, was a new concept. While ATiB's $50 \%$ annual turnover is more extreme than the typical organisation, modern firms, especially in the dot.com high tech world, are faced with the problem of preparing for the contingency of losing a key employee. ATiB has just begun to plan for this, but it appears that building redundancy among different students through mentorship will help to mitigate most adverse effects.

There is a clear relationship between problems of attrition and those of acclimating new workers. Industries characterised by high levels of worker mobility such as IT will be faced with both problems on an ongoing basis. Furthermore, trends towards 'free agency', the rise of the independent agent who sells some form of personal expertise, will make these problems more salient. Effective solutions to this pair of problems can provide substantial benefit for knowledge organisations.

Incorporating a knowledge audit into a knowledge management methodology. Alavi (2000) suggested two important issues for KM system design: 1) the development of methodologies for identifying factors that influence the knowledge management requirements of the firm and 2) understanding the relationship between knowledge requirements and KM model (repository or network). The knowledge audit has something to offer for both of these key questions. By forcing an articulation of knowledge goals, the knowledge audit moves stakeholders toward an understanding of organisational knowledge requirements. Knowledge audits and assessments are iterative activities. Thus the knowledge strategies as well as the generic knowledge processes undergo change as part of each iteration. Ongoing evaluation of requirements and the means of satisfying them are part and parcel of each iteration and are kicked-off by the audit.

Because of the contrasting ontological assumptions about knowledge, one may be tempted to regard the repository and network models as being incompatible, that a firm can opt for one or the other. Spender's (1996) model offers insight here. Knowledge that is social and explicit is amenable to codification and storage in a repository. Knowledge that is social and tacit is the basis for organisational culture, values, and beliefs. The network model enhances the exchange of this type of knowledge. The ATiB Portal is an attempt to support both models by storing organisational/explicit knowledge in a repository and by enhancing and enlarging existing networks through discussion groups and providing extensive contact information.

\section{REFERENCES}

Ackerman, M.S. (1996). Definitional and contextual issues in organizational and group memories, Information Technology and People, 9, 1, 10-24.

Agarwal, R., Kannar, R, and M. Tanniru, "Formal Validation of a Knowledge-Based System Using a Variation of the Turing Test", Expert Systems with Applications, 1993, Vol.6, pp.181-192

Alavi, Maryam (2000). Managing organizational knowledge in Framing the Domains of IT Management, Robert W. Zmud Ed., Cincinnati: Pinnaflex Educational Resources, Inc.

Alavi, M and D. Leidner (2001). Conceptual foundations of knowledge management and knowledge management systems, MIS Quarterly.

Cook, Scott D. N., Brown, John Seely (1999). Bridging Epistemologies: The Generative Dance Between Organizational Knowledge and Organizational Knowing, Organization Science, 10, 4, 381-400. 
Croasdell, David T. (2001). IT's role in organizational memory and learning, Information Systems Management, $18,1,8-11$.

Davenport, Thomas H., Jarvenpaa, Sirkka L., Beers, Michael C. (1996). Improving Knowledge Work Processes, Sloan Management Review, 36, 53-65, Summer, 1996.

Davenport, Thomas H. (1999). Knowledge Management and the Broader Firm: Strategy, Advantage, and Performance, in Knowledge Management Handbook, Jay Liebowitz, ed., Boca Raton, FL: CRC Press.

Dierick, I. and K. Cool (1989). Asset stock accumulation and sustainable competitive advantage, Management Science, 35, 12, $1504-1514$.

Drucker, Peter F. (1978). The Age of Discontinuity, Harper \& Row: New York.

Drucker, Peter F. (1999). Knowledge-Worker Productivity: The Biggest Challenge, California Management Review, 41, 2, 79 - 94.

Duffy, Jan (2000). The KM Infrastructure, Information Management Journal, 34, 2, 62 - 66.

Fahey, L. and L. Prusak (1998). The eleven deadliest sins of knowledge management, California Management Review, 40, 3, $265-280$.

Grant, Robert M. (1996). Toward a Knowledge-Based Theory of the Firm, Strategic Management Journal, 7 , Winter Special Issue, 109-122.

Hammer, Michael and Champy, James (1993). Reengineering the Corporation, New York: HarperCollins Publishers

Hansen, M.T., N. Nohira, and T. Tiemey (1999). What's your strategy for managing knowledge? Harvard Business Review. March-April 1999, 106 - 116.

Kettinger, William J., Teng, James T. C., and Guha, Subashish (1997). Business Process Change: A Study of Methodologies, Techniques, and Tools, MIS Quarterly, 21, 1, 55-80.

Klein, Mark M. (1994). Reengineering Methodologies and Tools, Information System Management, Spring 1994

Leavitt, Harold J. (1972). Managerial Psychology: An Introduction to Individuals, Pairs, and Groups in Organizations, Chicago: University of Chicago Press.

Lippmann, S. A. and R. P. Rumelt (1982). Uncertain imitability: an analysis of interfirm differences in efficiency under competition, Bell Journal of Economics, 13, $418-438$.

Nonaka, I. (1994). A dynamic theory of organizational knowledge creation, Organizational Science, 5, 1, $14-37$.

Nonaka, I. and Takeuchi, H. (1995). The Knowledge Creating Company, New York: Oxford University Press.

Pfeffer, Jeffrey, Sutton, Robert I. (1999). Knowing "What" to Do Is Not Enough: Turning Knowledge into Action, California Management Review, 42, 1, 83-108.

Polyani, M. (1967). The tacit dimension. London: Routledge and Kegan Paul.

Polyani, M. (1962). Personal knowledge: toward a post-critical philosophy. New York: Harper Torchbooks.

Probst, Gilbert, Raub, Steffen, Romhardt, Kai (2000). Managing Knowledge: Building Blocks for Success, Chichester: John Wiley \& Sons Ltd.

Rummler, G. and Brache, A. (1995). How to Manage the White Space on the Organization Chart (2nd ED.), New York: Jossey Bass, Inc.

Sena, James A., Shani, A. B. (Rami) (1999). Intellectual Capital and Knowledge Creation: Towards an Alternative Framework, in Knowledge Management Handbook, Jay Liebowitz, ed., Boca Raton, FL: CRC Press.

Spender, J.-C. (1996). Making Knowledge the Basis of a Dynamic Theory of the Firm, Strategic Management Journal, 7, Winter Special Issue, 45-62.

Winter, S. G. (1995). Four Rs of profitability: rents, resources, routines, and replications, in Resource-based and evolutionary theories of the firm: towards a synthesis, C. A. Montgomery ed. Norwell, MA: Kluwer, pp. 147 $-178$. 